\title{
Structural Changes in the Tracheae of Preterm Lambs Induced by Ventilation
}

\author{
KIRAN S. DEORAS, MARLA R. WOLFSON, VINOD K. BHUTANI, AND THOMAS H. SHAFFER \\ Departments of Physiology and Pediatrics, Temple University School of Medicine, \\ Philadelphia, Pennsylvania 19140 and Section on Newborn Pediatrics, Pennsylvania Hospital, \\ Philadelphia, Pennsylvania 19107
}

\begin{abstract}
Compliant immature airways sustain significant deformation following positive pressure ventilation. To evaluate the structural changes induced by in vivo positive pressure ventilation, tracheae of preterm lambs (107-116 d gestational age) were studied histologically. Nonventilated (group I: $n=7$ ) and ventilated (group II: $n$ $=7$ ) tracheal segments were excised and studied by histologic and morphometric techniques. Computerized image analysis was used to measure dimensions of tracheal wall components and of the tracheal section. The circumference, diameter, and cross-sectional area of the section as well as the length of the trachealis muscle were significantly greater; although the thickness of the muscle and cartilage were seen to be significantly lower in group II sections compared to group I sections. Also, in comparison to group I, in group II sections there was lesser overlap of the posterior free ends of tracheal cartilage and the epithelial layer was flattened and focally abraded. Our findings demonstrate structural changes in the airway of preterm animals and characterize alterations in the geometric arrangement of muscle and cartilage after PPV. These results suggest possible structural mechanisms for the functional changes seen during and subsequent to mechanical ventilation. (Pediatr Res 26: 434-437, 1989)
\end{abstract}

\section{Abbreviation}

PPV, positive pressure ventilation

The airways of immature animals are known to be very compliant, collapsible structures when compared to those of more mature animals (1-4). PPV further increases airway collapsibility, decreases the inflationary complicance (5), produces chronic volume deformation (6-8), and results in airway epithelial damage (9-14). To date, insufficient data are available to identify the morphologic alterations in the principal components of the tracheal wall (cartilage and smooth muscle) after positive pressure ventilation. Structural changes within the tracheal wall may explain, in part, the mechanical alterations consequent to ventilation. To test the hypothesis that PPV produces structural changes in the tracheal cartilage and smooth muscle dimensions, both ventilated and unventilated tracheal segments were excised from premature lambs and examined by histologic and morphometric techniques. Potential differences in muscle and cartilage

Received January 31, 1989; accepted July 13, 1989.

Correspondence Kiran S. Deoras MBBS, Ph.D., \% Thomas H. Shaffer, Ph.D., Department of Physiology, Temple University, School of Medicine, 3420 N. Broad Street, Philadelphia, PA 19140.

Supported by NIH Grant HL 32031. dimensions were analyzed for statistical significance between the ventilated and unventilated groups.

\section{MATERIALS AND METHODS}

This study used tracheal segments obtained from age- and wtmatched preterm lambs between 107 and 116 d gestational age. The protocols for these studies had been approved by the Institutional Animal Care and Use Committee of Temple University, Philadelphia.

Animal preparation. Pregnant ewes of known gestation were restrained in a prone position after sedation (Ketamine $\mathrm{HCl}, 500$ $\mathrm{mg}$ intramuscular) and epidural anesthesia $(0.5-1 \mathrm{mg} / \mathrm{kg}, 0.75 \%$ bupivacaine $\mathrm{HCl}$ ). The uterus was exposed ventrolaterally and the fetal head allowed to emerge through a uterotomy. A rubber glove filled with warm saline was promptly placed over the head to prevent spontaneous inspiration of air. Under local anesthesia $(4 \mathrm{mg} / \mathrm{kg}, 0.5 \%$ lidocaine $\mathrm{HCl}$ ) the fetal right carotid artery and jugular vein were cannulated. The trachea was also cannulated midway along its length with a close fitting flurocarbon filled, proximally occluded tracheal tube. After delivery, the lambs were immediately ventilated with warm oxygenated liquid fluorocarbon $\left(\mathrm{FiO}_{2}\right.$ : 1 ; mean airway pressure: $5-12 \mathrm{~cm} \mathrm{H}_{2} \mathrm{O}$; frequency: five to eight breaths/min; duration: $2-3$ hours) as described by Wolfson et al. (15). Arterial blood gas tensions, pH, blood pressure, and the ECG were monitored throughout the experiment to maintain cardiopulmonary stability. According to protocol, the animals were sacrificed with intravenous T61 (HoechstRoussel Agri-Vet Co., Somerville, NJ) after approximately $2 \mathrm{~h}$ and tracheal segments excised immediately upon death.

Unventilated segments (group I, $n=7$ ) were obtained from a portion of the trachea cranial to the tracheostomy, whereas ventilated segments (group II, $n=7$ ) were obtained from the portion of the trachea caudal to the tracheostomy. Care was taken to ensure that the excised tracheal segments were not in direct contact with the tracheostomy tube. All segments were excised from a region of the trachea whose borders were at least $3 \mathrm{~mm}$ below the cricoid cartilage and $20 \mathrm{~mm}$ above the carina. Previous measurements of untreated segments have shown that the tracheal dimensions do not change significantly throughout this region and that the trachea can be regarded as a cylinder ( 4 , $6,7)$.

Morphology and morphometry. The excised tracheal segments were routinely prepared for histologic examination (16) as follows: fixed in $2 \%$ cold glutaraldehyde in phosphate buffer, dehydrated through varying concentrations of alcohol, cleared in cedarwood oil, and embedded in paraffin. Sections of $7 \mu \mathrm{m}$ were cut serially with a microtome, mounted on glass slides and stained with Milligan's Trichrome stain, which presented a strong contrast between muscle and cartilage and facilitated differentia- 
tion between the principal tracheal wall components. The slides were examined by light microscopy and used for morphometric measurements. Selected sections were photographed on Kodak Ektachrome 5077 color slide film (Eastman Kodak, Rochester, NY) with a dissecting microscope. Histologic analyses were performed by an investigator unaware of the mode of treatment of the tracheal segments.

A computerized image analysis system $(17,18)$ was used for the morphometric measurements (courtesy Dr. J. B. Sheffield, Department of Biology, Temple University, Philadelphia, PA). The system is equipped to conduct planimetric measurements with a program to compute morphologic and densitometric parameters of a user selected and outlined region.

Slides prepared for light microscopy were used for conducting the morphometric measurements. These were placed under a light microscope, digitized, and presented on a video monitor coupled to a touch screen. The outlines of the different parameters to be measured were traced onto the touch screen. Length, area, and thickness of the tracheal cartilage and trachealis smooth muscle fibers were measured as were the diameter, circumference, and cross-sectional area of each tracheal section. Figure 1 is a diagrammatic representation of the tracheal cross-section and depicts the various parameters measured.

Tracheal injury was also evaluated using the histopathologic scoring system described by Ophoven et al. (12), according to which, nine variables reflecting pathologic changes in mucosal surface tissues are examined and graded in four levels according to severity. Thus, the transverse tracheal sections were examined for the absence/presence and severity of: epithelial erosion, necrosis, submucosal infiltration of polymorphonuclear leukocytes, surface ciliary loss, loss of intraepithelial mucus, luminal debris, hemorrhage, edema, and hyperplasia.

Statistical analysis. Groups I and II were matched for gestational age and body wt. Intergroup differences in tracheal cartilage and muscle length and thickness, mean tracheal diameter, circumference, cross-sectional area, and histopathologic score were analyzed for significance using the Student's $t$ test for independent samples. Statistical significance was accepted at the $p<0.05$ level. All values are denoted as mean \pm SEM.

\section{RESULTS}

Preterm lambs supported by ventilation with oxygenated liquid fluorocarbon were biologically stable as determined by gas exchange and acid-base data $\left(\mathrm{PO}_{2}>100 \mathrm{~mm} \mathrm{Hg} ; \mathrm{PCO}_{2}: 35-45\right.$ $\mathrm{mm} \mathrm{Hg}$; $\mathrm{pH}$ : 7.25-7.45). The heart rate and mean arterial pressure also remained stable during ventilation (duration $\sim 2 \mathrm{~h}$ ).

Dimensions and histopathologic scores of the tracheal sections

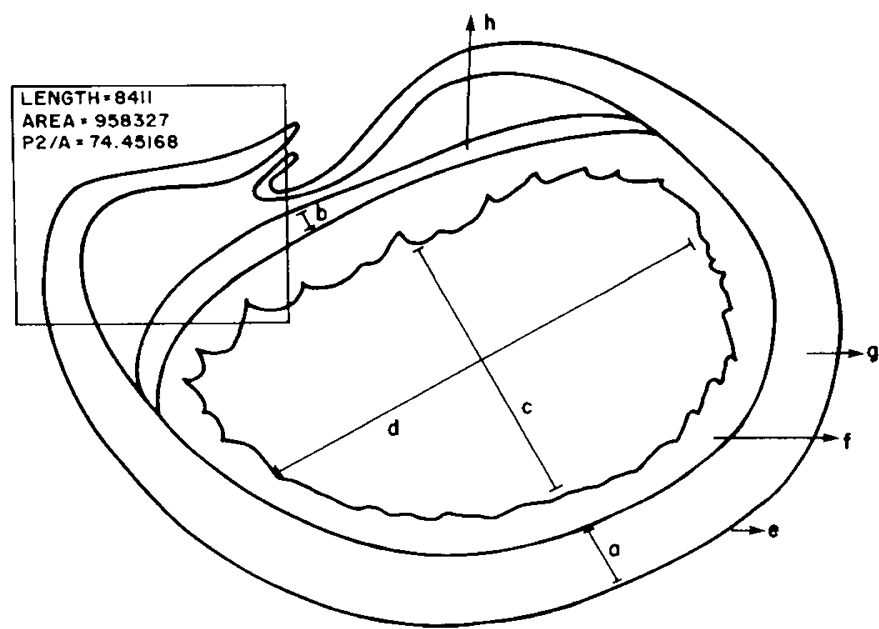

Fig. 1. Diagrammatic representation of a tracheal cross-section depicting the various parameters measured. $a$, cartilage thickness; $b$, muscle thickness; $c$, anteroposterior diameter; $d$, transverse diameter; $e$, external circumference; $f$, internal circumference; $g$, cartilage length; and $h$, muscle length. The inset shows dimensions; length, area, and perimeter ${ }^{2} /$ area (in pixels) of the enclosed portion of cartilage.

Table 1. Tracheal dimensions*

\begin{tabular}{|c|c|c|c|c|c|c|c|c|c|}
\hline Experiment & $\begin{array}{l}\text { DA } \\
\text { (d) }\end{array}$ & $\begin{array}{c}\mathrm{ML} \\
(\mathrm{mm})\end{array}$ & $\begin{array}{c}\mathrm{CL} \\
(\mathrm{mm}) \\
\end{array}$ & $\begin{array}{c}\text { DIA } \\
(\mathrm{mm})\end{array}$ & $\begin{array}{c}\mathrm{CSA} \\
\left(\mathrm{mm}^{2}\right) \\
\end{array}$ & $\begin{array}{c}\text { I.CIRC } \\
(\mathrm{mm})\end{array}$ & $\begin{array}{c}\mathrm{CT} \\
(\mathrm{mm}) \\
\end{array}$ & $\begin{array}{c}\mathrm{MT} \\
(\mathrm{mm}) \\
\end{array}$ & $\begin{array}{c}\mathrm{HP} \\
\text { score }\end{array}$ \\
\hline \multicolumn{10}{|c|}{ Group I: unventilated tracheal dimensions } \\
\hline 1 & 107 & 6.36 & 19.46 & 4.00 & 17.58 & 12.56 & 0.60 & 0.28 & 0 \\
\hline 2 & 107 & 5.51 & 17.69 & 3.55 & 15.29 & 11.15 & 0.56 & 0.14 & 0 \\
\hline 3 & 114 & 6.06 & 17.07 & 4.00 & 13.95 & 12.56 & 0.55 & 0.27 & 0 \\
\hline 4 & 115 & 5.90 & 21.00 & 3.80 & 15.01 & 11.93 & 0.73 & 0.39 & 0 \\
\hline 5 & 115 & 4.40 & 20.10 & 3.50 & 14.43 & 10.99 & 0.61 & 0.43 & 0 \\
\hline 6 & 115 & 5.54 & 20.86 & 3.88 & 15.43 & 12.18 & 0.55 & 0.29 & 0 \\
\hline 7 & 116 & 5.46 & 20.07 & 3.75 & 13.62 & 12.47 & 0.54 & 0.39 & 0 \\
\hline Mean & 113 & 5.61 & 19.46 & 3.78 & 15.04 & 11.98 & 0.59 & 0.31 & 0 \\
\hline $\mathrm{SE} \pm$ & 1 & 0.24 & 0.58 & 0.08 & 0.49 & 0.25 & 0.03 & 0.04 & 0 \\
\hline \multicolumn{10}{|c|}{ Group II: ventilated tracheal dimensions } \\
\hline 1 & 107 & 7.42 & 15.89 & 5.00 & 18.65 & 15.70 & 0.56 & 0.10 & 4 \\
\hline 2 & 107 & 6.91 & 15.79 & 4.50 & 16.65 & 14.13 & 0.42 & 0.19 & 5 \\
\hline 3 & 114 & 6.38 & 17.31 & 5.25 & 15.50 & 14.80 & 0.42 & 0.10 & 4 \\
\hline 4 & 115 & 6.64 & 19.17 & 4.50 & 17.46 & 16.12 & 0.59 & 0.16 & 4 \\
\hline 5 & 115 & 5.68 & 16.06 & 3.85 & 15.24 & 13.09 & 0.52 & 0.12 & 5 \\
\hline 6 & 115 & 7.12 & 19.81 & 6.00 & 16.38 & 15.82 & 0.47 & 0.22 & 4 \\
\hline 7 & 116 & 6.99 & 19.95 & 5.25 & 20.91 & 16.49 & 0.54 & 0.25 & 5 \\
\hline Mean & 113 & $6.73 \dagger$ & 17.71 & $4.91 \dagger$ & $17.26 \dagger$ & $15.16 \dagger$ & $0.50 \dagger$ & $0.16 \dagger$ & $4.43 \dagger$ \\
\hline $\mathrm{SE} \pm$ & 1 & 0.22 & 0.71 & 0.26 & 0.75 & 0.46 & 0.03 & 0.02 & 0.19 \\
\hline
\end{tabular}

* DA, developmental age in days; ML, muscle length; CL, cartilage length; DIA, diameter; CSA, cross-sectional area; I.CIRC, internal circumference; $\mathrm{CT}$, cartilage thickness; MT, muscle thickness; HP score, histopathologic score.

$$
\dagger p<0.05 \text {. }
$$


for both the unventilated (group I) and ventilated (group II) segments are presented in Table 1 .

In sections obtained from the ventilated segments, the tracheal cross-sectional area, circumference, and mean diameter were found to be significantly greater when compared to sections obtained from the unventilated segments. The greater circumference was mainly due to the greater length of the trachealis muscle in these sections, whereas the cartilage length remained essentially unchanged. Mean cartilage thickness and muscle thickness were also found to be significantly less in the ventilated sections compared to the unventilated sections.

Upon microscopic examination, the sections from the unventilated segments showed the cartilagenous arcs with posterior overlapping free ends and the intact epithelial lining thrown into folds. Whereas the sections from the ventilated segments showed an absent or greatly reduced overlap of the posterior free ends of cartilage and an extremely thinned and stretched trachealis muscle. Representative sections from the two groups are depicted in Figures $2 a$ and $b$.

In sections from the ventilated segments, closer histologic examination showed areas of focal epithelial erosions affecting $15-50 \%$ of the tracheal circumference (Fig. 3), associated with scattered loss of surface cilia and occasional absence of mucuscontaining goblet cells. Focal scattered infiltration of polymorphonuclear leukocytes and minimal submucosal hemorrhage

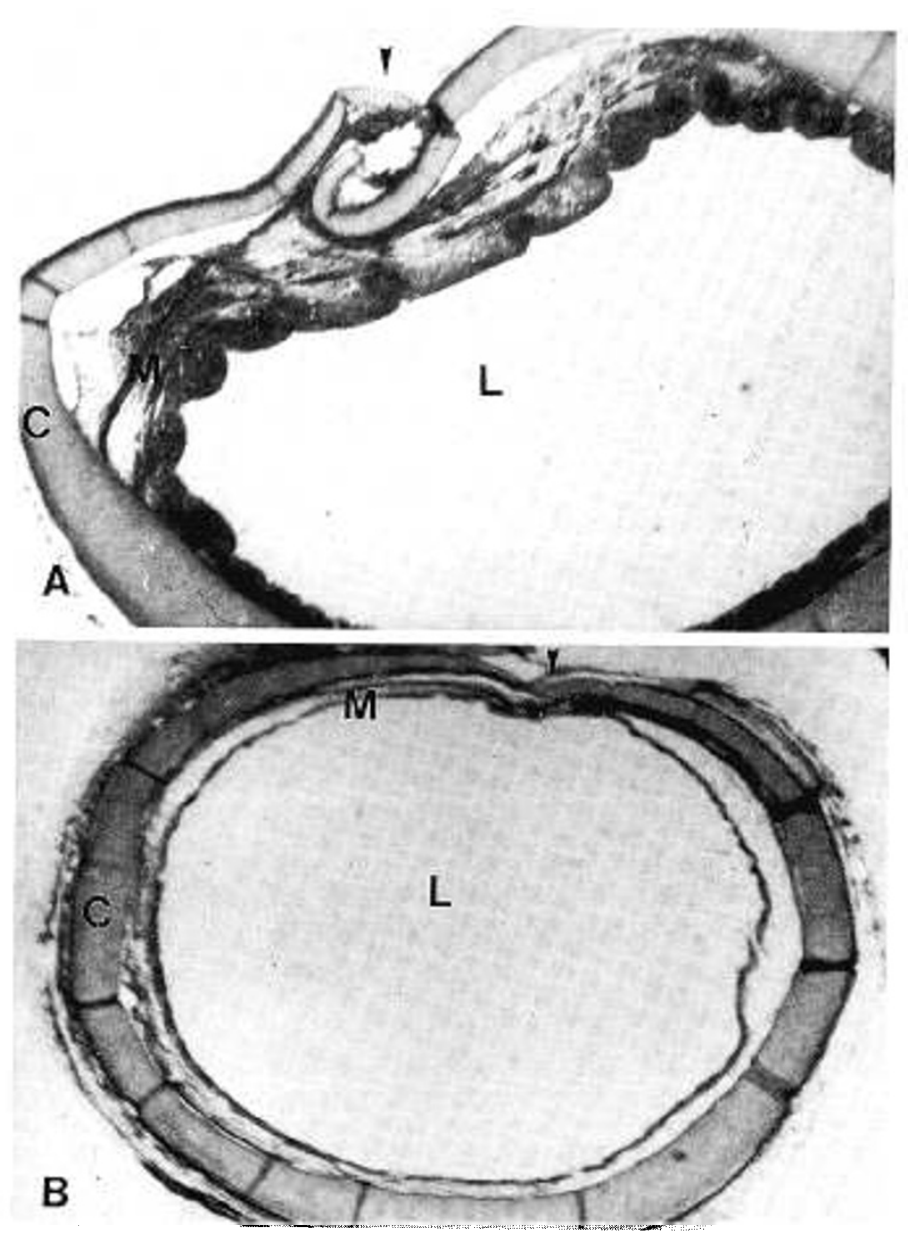

Fig. 2. $A$, representative section from an unventilated tracheal segment. The entire tracheal ring with intact epithelium and overlapping ends of cartilage in the posterior wall (arrow) are seen $(C$, cartilage; $M$, muscle; and $L$, lumen. $30 \times$ ). $B$, representative section from a ventilated tracheal segment. Note the thinned smooth muscle fibers and epithelium; and no overlap of the free ends of cartilage in the posterior wall (arrow) ( $C$, cartilage; $M$, muscle; and $L$, lumen. $30 \times$ ).

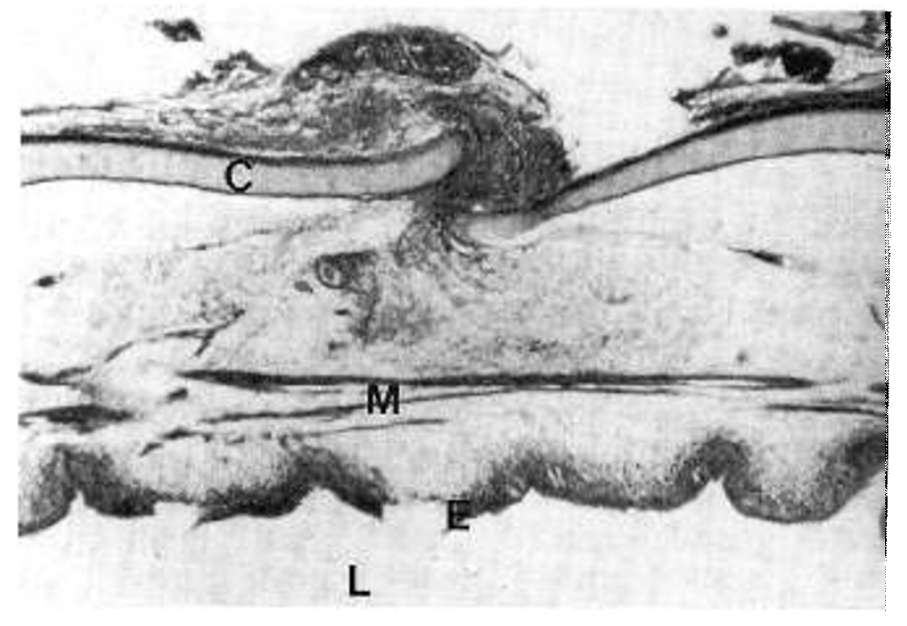

Fig. 3. Transverse section of the trachea from a ventilated segment depicting the abraded epithelial surface $(C$, cartilage; $M$, muscle; $L$, lumen; and $E$, epithelium. $60 \times$ ).

were also seen. No edema or hyperplasia were noted, nor was any luminal debris seen.

The histologic injury scores for the unventilated tracheal segments were uniformly zero. Airway injury was seen in all the ventilated segments. The histologic injury scores for both groups of animals are compared in Table 1.

\section{DISCUSSION}

These experiments used tracheae from preterm lambs ranging in age from 107 to $116 \mathrm{~d}$ of gestation, appropriately oxygenated and ventilated in vivo with liquid fluorocarbon. Cardiopulmonary stability of these preterm lambs with respect to oxygenation, $\mathrm{CO}_{2}$ elimination, acid-base, and hemodynamic status was achieved using liquid ventilation support, as this is the only means available currently to ventilate these very premature lambs $(15,19)$. It is important to emphasize the necessity of achieving gas exchange/acid base status within physiologic limits as inability to do so could lead to histochemical damage of the airways, in turn affecting airway structure and function. In vivo ventilation takes into account the maintainence of intact local and central neural mechanisms, blood flow and the surrounding supporting structures.

Our observations suggest that the structural alterations consequent to ventilation are a result of barotrauma, and that pressure induced deformation is responsible for these changes (6). Studies by Bhutani and Shaffer (20) and Alsberge et al. (21) have demonstrated similar alterations in tracheal dimensions with both modes-liquid and conventional gas ventilation-when similar peak ventilatory pressures and durations were used. High frequency jet ventilation has also been shown to produce comparable mechanical alterations (22).

Previous studies have investigated the extent of airway barotrauma associated with positive pressure ventilation by examining a variety of parameters viz. airway function $(5,6)$, dimensions $(7,8)$, and epithelial damage (9-14). In this study, our interest focused on the effect of positive pressure ventilation on the principal tracheal wall components, the trachealis muscle, and the tracheal cartilage, since changes in the dimensions or geometry of these components may provide an explanation for the previously documented alterations in airway function.

Positive pressure ventilation has been shown to cause changes in the functional characteristics of the trachea, which include a decrease in its inflationary compliance and an increase in its collapsing compliance $(5,6)$. This bidirectional change in compliance characteristics suggests the possibility of multiple structural alterations being responsible for the functional changes.

When relaxed tracheae are subjected to a negative transmural 
pressure they exhibit invagination of their posterior membranous wall. This is associated with a reduction in intraluminal volume, the magnitude of which is determined by the organ's collapsing compliance. Our results demonstrate a significant increase in the muscle length and a decrease in muscle thickness after PPV. A longer and thinner muscle contributes to a posterior wall that is subject to greater invagination at a given negative transmural pressure. The greater muscle length and associated decrease in the overlap of the posterior free ends of cartilage, therefore, render the trachea more vulnerable to pressure induced deformation and collapse.

Our results demonstrate increases in tracheal cross-sectional area, diameter and circumference, and are indicative of pressure induced deformation following PPV. These findings are consistent with those of Bhutani et al. (7) who showed radiographic evidence of tracheomegaly in preterm infants after a period of mechanical ventilation.

Airway epithelial damage and the occurrence of necrotizing tracheobronchitis following assisted ventilation has been studied in various animal models. The extent of damage to the airway epithelium seen on light microscopy in the current study, with mean injury scores of four, is consistent with the findings of Ophoven et al. (12). Furthermore, our findings correspond to the acute inflammatory pattern of histopathologic injury commonly seen after conventional mechanical ventilation (12). This type of damage with focal epithelial erosions, has also been reported by Lee and O'Brodovich (10) and Polak et al. (13) for human infants after assisted conventional PPV. Various factors have been implicated in the etiology of airway epithelial damage including barotrauma, high ambient oxygen tension, inadequate humidification, ventilatory frequency, inflammation, and physical factors such as shear stress (9-14). We used warm liquid fluorocarbon as the ventilating medium at a frequency of five to eight breaths/min, thus eliminating inadequate humidification and high respiratory frequency as causative factors. However, multiple determinants such as barotrauma, high ambient oxygen tension, and physical factors cannot be excluded. The respiratory epithelium is known to release relaxant substances (e.g. epithelial derived relaxant factor) that alter the response of the tracheal smooth muscle to constrictor and relaxant agents (23). We speculate, that the loss of portions of the epithelium, as seen after PPV, could lead to decreased relaxation or increased contraction of the tracheal smooth muscle thus contributing to the lower inflationary compliance seen after PPV. Although this may be one mechanism to explain the change in compliance, other vasoactive and bronchoreactive substances such as platelet-activating factor, prostanoids, etc., released either from the injured epithelium or the surviving cells may contribute to the functional changes seen after PPV.

In summary, our findings show various changes in the structural characteristics of the tracheal wall components after PPV and associated barotrauma. All these factors considered together may help explain the concommitant changes in tracheal functional properties.
Acknowledgments. The authors thank Dr. J. B. Sheffield for his advice and assistance in the use of the computerized image analysis system, and Robert Roache for technical assistance.

\section{REFERENCES}

1. Bhutani VK, Rubenstein SD, Shaffer TH 1981 Pressure-volume relationships of trachea in fetal, neonatal and adult rabbits. Respir Physiol 43:221-231

2. Burnard ED, Grattan-Smith P, Picton-Marlowe CG, Grauaug A 1965 Pulmonary insufficiency in prematurity. Aust Pediatr J 1:12-38

3. Croteau JR, Cook CD 1961 Volume-pressure and length-tension measurements in human tracheal and bronchial segments. J Appl Physiol 16:170 172

4. Shaffer TH, Bhutani VK, Wolfson MR, Tran NN 1988 In vivo mechanical properties of the developing airway. Pediatr Res 25:143-146

5. Penn RB, Wolfson MR, Shaffer TH 1988 Effect of ventilation on immature airways. Pediatr Res 23:519-524

6. Bhutani VK, Rubenstein SD, Shaffer TH 1981 Pressure-induced deformation in immature airways. Pediatr Res 15:829-832

7. Bhutani VK, Ritchie WG, Shaffer TH 1986 Acquired tracheomegaly in very preterm neonates. Am J Dis Child 140:449-452

8. Palmer J, Schidlow D, Wolfson B, Capitanio M, Huang N 1979 Tracheomegaly: a finding in two children who survived prolonged intubation and mechanical ventilation for hyaline membrane disease. Pediatr Res 13:1282(abstr)

9. Clark RH, Wiswell TE, Null DM, deLemos RA, Coalson JJ 1987 Tracheal and bronchial injury in high frequency oscillatory ventilation compared with conventional positive pressure ventilation. $J$ Pediatr 111:114-118

10. Lee RMKW, O'Brodovich HM 1988 Airway epithelial damage in premature infants with respiratory failure. Am Rev Respir Dis 137:450-457

11. Mammel MC, Ophoven JP, Lewallen PK, Gordon MJ, Sutton MC, Boros SJ 1986 High frequency ventilation and tracheal injuries. Pediatrics 77:608613

12. Ophoven J, Mammel M, Gordon M, Boros S 1984 Tracheobronchial histopathology associated with high frequency ventilation. Crit Care Med 12:829832

13. Polak MJ, Donnelly WH, Bucciarelli RL 1989 Comparison of airway pathologic lesions after high-frequency jet or conventional ventilation. Amer J Dis Child 143:228-232

14. Wiswell TE, Clark RH, Null DM, Kuehl TJ, deLemos RA, Coalson JJ 1988 Tracheal and bronchial injury in high-frequency oscillatory ventilation and high-frequency flow interruption compared with conventional positive pressure ventilation. J Pediatr 112:249-256

15. Wolfson MR, Tran N, Bhutani VK, Shaffer TH 1988 A new experimental approach for the study of cardiopulmonary physiology during early development. J Appl Physiol 65:1436-1443

16. Humason GL 1979 Animal Tissue Techniques. W. H. Freeman and Co. San Francisco, pp 38-387

17. Sheffield JB, Hilfer SR, Silage D 1987 A simple image analysis system using a microcomputer and video grabber board. J Cell Biol 105:513a

18. Silage DA, Gil J 1984 The use of touch-sensitive screen in interactive morphometry. J Microscopy 134:315-321

19. Shaffer TH, Tran NN, Bhutani VK, Sivieri EM 1983 Cardiopulmonary function in very preterm lambs during liquid ventilation. Pediatr Res 17:680684

20. Bhutani VK, Shaffer TH 1983 Effect of liquid ventilation on preterm tracheal mechanics. Biol Neonate 44:257-263

21. Alsberge MS, Wolfson MR, Bhutani VK, Minczak BM, Shaffer TH 1987 Ventilation induced changes in the functional characteristics of immature airways. Fed Proc 46:513(abstr)

22. Bhutani VK, Shaffer TH, Spitzer AR, Fox WW 1986 Effect of high frequency jet ventilation on neonatal tracheal mechanics. Pediatr Pulmonol 1:327-33i

23. Cuss FM, Barnes PJ 1987 Epithelial mediators. Am Rev Respir Dis 136:832835 